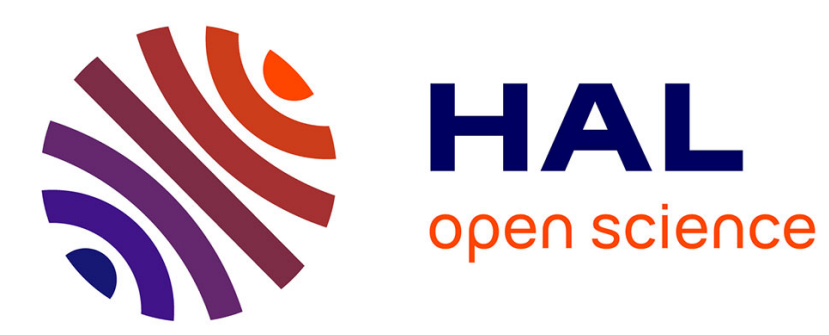

\title{
Hopper 2013/Cannes 2013 au prisme des écritures numériques
}

Emmanuel Ethis, Marie-Sylvie Poli

\section{To cite this version:}

Emmanuel Ethis, Marie-Sylvie Poli. Hopper 2013/Cannes 2013 au prisme des écritures numériques.

Culture et Musées, 2014, 24, pp.63-88. 10.4000/culturemusees.624 . hal-03262862

\section{HAL Id: hal-03262862 \\ https://hal.science/hal-03262862}

Submitted on 16 Jun 2021

HAL is a multi-disciplinary open access archive for the deposit and dissemination of scientific research documents, whether they are published or not. The documents may come from teaching and research institutions in France or abroad, or from public or private research centers.
L'archive ouverte pluridisciplinaire HAL, est destinée au dépôt et à la diffusion de documents scientifiques de niveau recherche, publiés ou non, émanant des établissements d'enseignement et de recherche français ou étrangers, des laboratoires publics ou privés. 


\section{OpenEdition}

Journals

\section{Culture \& Musées}

Muséologie et recherches sur la culture

24 | 2014

Démocratisation culturelle et numérique

\section{Hopper 2013/Cannes 2013 au prisme des écritures numériques}

Hopper 2013/Cannes 2013 through the lens of digital writing

Hopper 2013/Cannes 2013 en el prisma de las escrituras digitales

Emmanuel Ethis et Marie-Sylvie Poli

p. $63-88$

https://doi.org/10.4000/culturemusees.624

\section{Résumés}

\section{Français English Español}

Les événements culturels les plus importants se déclinent désormais avec force sur le Web et les réseaux sociaux. Le numérique permet d'amplifier l'espace-temps de l'événement lui-même tout en apportant un registre de participation plus immédiate. En choisissant durant la même année 2013 de rendre compte d'une diversité d'écritures numériques suscitées par deux dispositifs événementiels à large portée - l'exposition Edward Hopper à Paris et le Festival de Cannes -, il s'agit ici d'explorer des formes d'énonciation propres au numérique. Écriture contrainte par une image institutionnelle, écriture contrainte par le dispositif numérique lui-même, comme c'est le cas sur Twitter, écriture contrainte par la participation ouverte. On découvre ici une écriture participante tant institutionnelle, critique que publique, qui renforce, via l'espace de nouvelles " trivialités ", l'appropriation et l'existence sociale même de formes culturelles jusque-là circonscrites dans l'espace et dans le temps ainsi que les modalités par lesquelles s'inventent et se réinventent nos formes de médiation de la culture.

Major cultural events have now a growing visibility on the internet and social media. Digital technology magnifies the space-time spectrum of the event, while allowing users to participate with more immediate effect. Choosing two major and widely renowned events: the Edward Hopper exhibition in Paris and The Cannes Movie Festival in the same year of 2013 was a mean of exploring communication only digital technology can provide. Thus, scrutinizing a variety of digital writing was a way to decipher the constraints of the institutional image and of the digital tool itself. Twitter, for that matter is constrained to open participation. Reinforced via space and new forms of trivialities lies an institutional, critical as well as public participatory for writing, which reinforces the appropriation of the social existence of cultural forms, until then restricted space and timewise as well as the methods by which the mediation forms of culture create and recreate. 
De aquí en adelante, los eventos culturales de mayor importancia se gatillan desde la web y las redes sociales. Lo digital permite amplificar el espacio tiempo del acontecimiento en sí, permitiendo un registro de participación más inmediato. Al dar cuenta durante el mismo año 2013 de una diversidad de escrituras digitales suscitadas por dos eventos de largo aliento como dispositivos (la exposición Edward Hopper en parís y el Festival de Cannes), se trata aquí de explorar las formas de enunciación propias de lo digital. Escritura constreñida por una imagen institucional, escritura constreñida por el dispositivo digital en sí como el caso de Twitter, escritura constreñida por la participación abierta. Descubrimos aquí una escritura participante, tanto institucional como crítica y pública que refuerza vía el espacio nuevas trivialidades, la apropiación y la existencia social, aún de formas culturales hasta ahí circunscritas al espacio y al tiempo así como a las modalidades por las cuales se inventan y se reinventan nuestras formas de mediación de la cultura.

\section{Entrées d'index}

Mots-clés : communication, numérique, événement, écriture, festival, exposition

Keywords: communication, digital, events, writing, festival, exhibition

Palabras clave: comunicación, digital, acontecimiento, escritura, festival, exposición

\section{Notes de la rédaction}

Manuscrit reçu le : 17 décembre 2013

Version révisée, reçue le : 23 juillet 2013

2e version révisée, reçue le : 14 août 2013

Article accepté pour publication le : 3 septembre 2014

\section{Texte intégral}

Voilà une dizaine d'années déjà que la recherche en sciences de l'information et de la communication, mettant à profit les avancées de Jack Goody qui nous a démontré que toute écriture est à la fois une technique et la condition de la pensée (Goody, 1994), aborde l'écriture numérique à partir de ses dispositifs sémiotiques, technologiques et symboliques (Souchier, 2012 ; Jeanneret, 2007 ; Jouët, 2001 ; Brockmeier, 2012).

2 Durant cette même période, d'autres chercheurs ont développé des approches interdisciplinaires qui ont permis de renouveier les travaux fondateurs des philosophes (Hall, 2007) et des sociologues de la culture (Becker, 1988) sur la problématique des publics de la culture (Eidelman, 2010), à partir d'une approche pius pragmatique et communicationnelle (Ethis, 2009 ; Poli \& Ancel, 2014) que socio-politique (Bourdieu, 1979). Mais ce n'est que récemment que des projets radicalement interdisciplinaires ${ }^{1}$ mobilisent des informaticiens et des chercheurs en sciences humaines et sociales sur des thèmes fédérateurs tels que culture, patrimoines et territoires, politiques culturelles (Gaillard, El Beze \& Ethis, 2013 ; Andreacola, Poli \& Sanjuan, 2013). Le moment est donc propice, pour nous qui analysons les expositions et les festivals en tant que " formes et modèles culturels ${ }^{2}$ », à l'étude des ressorts et des enjeux de l'écriture numérique de cette forme précise qu'est l'événement culturel, questionné depuis peu comme un dispositif communicationnel et culturel complexe (Merzeau, 2013 ; Paveau, 2013), dans un esprit de décioisonnement épistémologique et d'enrichissement méthodoiogique.

3 Souvent abordé comme un phénomène lié à l'avènement de l'industrie de la culture par les philosophes (Ricoeur, 1985) et les historiens de l'art (Clair, 2007), l'événement culturel est considéré par les médias comme une manifestation temporaire, une opération visant à renforcer ou améliorer l'image d'une institution, plutôt à destination du grand public : «L'événement est un fait lié à l'actualité qui est d'abord constitué comme information, qui est en train (ou qui va) de se passer et qui est considéré sur le moment par les journalistes comme suffisamment important pour faire l'objet d'une présentation particulière et valorisante. » (Champagne, 2000.) Il est considéré par les sociologues, les historiens et les sémioticiens comme une forme culturelle légitime à part entière, apparue autour des années soixante-dix dans les mondes de la culture au sens large (cinéma, arts vivants, 
musique, musées, patrimoines) : "Pour qu’il y ait événement il faut qu'il soit connu. » (Nora, 1972.) Ce qui explique notamment que les experts en sciences de gestion, en marketing ou en économie de la culture (Tobelem, 2010 ; Benhamou, 2012) s'y intéressent aussi de très près.

En revanche, l'« écriture numérique de l'événement culturel » que nous envisageons dans cet article comme un processus intermédiatique de traitement des informations (Gaudreault \& Jost, 2000), de création et d'échange de connaissances n'émerge que plus tard sur la Toile, au début des années 2000.

5 On assiste dès lors à la convergence entre, d'une part, l'augmentation du nombre de festivals et la multiplication des expositions temporaires (Jacobi, 2013) et, d'autre part, l'« explosion » des dispositifs d'écriture numérique désormais dédiés à l'expression des professionnels, des amateurs de culture, des lettrés du numérique (Doueihi, 2011), des internautes. Ces dispositifs d'échange des connaissances et des opinions sont tous liés, de près ou de loin, aux publics et aux usagers des mondes de la culture (articles, blogs, chats, critiques, réseaux sociaux) : "Immédiateté, instantanéité, volatilité, et toute la dimension éphémère de ce nouveau système de consignation qui force la création de nouvelles manières de trier, de préserver et de décrire. Le monopole des lettrés s'estompe. » (Caron, 2011 : 99.) Les questions théoriques posées par cette technique particulière d'écriture de l'événement culturel se posent également du côté de la réception, c'est-à-dire du point de vue de sa lecture et de sa mise en sociabilité par des usagers d'Internet dont on ne sait pas encore précisément quelles sont leurs intentions, leurs motivations et leurs connaissances sur l'émetteur du message lorsqu'ils " tombent " sur un article, un blog, un site ou un tweet à propos d'un événement culturel : " Il faudrait apprendre à recevoir l'information sans se laisser fasciner par ce qui est censé faire événement. » (Chardel \& Rockill, 2009 : 65.)

\section{L'écriture numérique de l'événement culturel sur le Web et les réseaux sociaux : choix méthodologique et problématique}

6 Cet article n'a pas pour ambition de traiter, en général, du concept d'écriture numérique. Notre objectif est d'analyser concrètement comment les écritures numériques qui circulent sur le Web participatif configurent leurs propres modes de communication des événements culturels. Ce parti pris implique de faire des choix, notamment de décider des événements culturels à retenir comme terrains d'analyse.

$7 \quad$ Afin de ne pas nous éloigner de nos objets de recherche privilégiés, nous avons décidé de retenir deux événements culturels récents, dotés d'une forte légitimité institutionnelle et culturelle, mais représentatifs de deux univers artistiques distincts, l'exposition de peinture et le cinéma. Il s'agit du Festival de Cannes et de l'exposition Hopper à Paris, ayant eu lieu tous deux en 2013. Bien que distincts à première vue - puisque les espaces qui leur étaient traditionnellement consacrés ne se recoupaient pas dans les médias qui leur étaient dévolus -, ces deux événements partagent des similitudes qui nous ont permis d'appréhender leur existence numérique sur la Toile à partir d'un même cadre d'analyse.

En premier lieu, ils sont confrontés à des contraintes de temporalités balisées par des dates de début et de fin ainsi qu'à des contraintes opératoires, matérielles, politiques et symboliques qui contribuent à structurer leurs publics. En deuxième lieu, lorsqu'il s'agit d'événements aussi médiatisés et reconnus comme économiquement rentables que le Festival de Cannes ou les expositions monographiques organisées au Grand Palais par la Réunion des musées nationaux (RMN), ils font l'objet d'une sorte de frénésie de 
commentaires écrits et transmédias, échangés sur les réseaux sociaux et les plateformes de médiations numériques avant, pendant et après qu'ils aient fait événement. Enfin, leur retentissement médiatique est amplifié grâce aux usagers du Web 2.0 et des réseaux sociaux qui, comme les professionnels de la culture, partagent désormais de facto les capacités d'écriture numérique nécessaires pour participer à la médiatisation de ces événements. D'autant que le discours du marketing culturel incite à jouer sans réserve cette carte de l'événementiel pour tenter une fois de plus de réaliser, grâce à l'écriture numérique, cet utopique franchissement des barrières de classes devant déboucher sur une démocratisation culturelle nourrie aux imaginaires des lettrés, des ingénieurs et des politiques (Chantepie \& Diberder, 2010).

9 Mais cette idéologie qui prône le numérique comme l'ultime révolution culturelle en cours s'inscrit-elle pour autant " dans un contexte d'universalisation des valeurs, des contenus, des marques et des imaginaires, sources nouvelles d'inspirations, et nouvelles scènes pour la société du spectacle et du storytelling, pour lesquelles les barrières sociales et anticorps ne suivent pas toujours le rythme effréné d'évolution » (Jutant, 2013:30) ? Ce questionnement, ou plutôt ce doute que nous partageons sur ce point, et qui fonde la problématique de l'article, ressort de travaux récents sur le numérique et la culture. Ces travaux invitent à considérer le numérique non pas comme une révolution technologique ou un nouveau paradigme culturel mais comme une nouvelle forme d'écriture qui permet des échanges et des interactions en tout lieu et à tout instant : "Qu'est-ce que le numérique, sinon une forme d'écriture ? Le numérique c'est l'écriture s'inscrivant et pouvant être "lue" par des automates, "scannée" à la vitesse de la lumière : c'est une écriture (et une lecture) essentiellement automatique. » (Stiegler, 2013 : 97.)

Car si, comme toute écriture de médiation culturelle, l'écriture numérique de l'événement culturel relève aussi d'une mécanique d'énonciation du subjectif, de l'évaluatif et du critique, elle est différente des autres écritures de l'événement culturel - non numériques donc - parce qu'elle est en lien direct et permanent avec l'architecture de l'Internet qui constitue désormais l'infrastructure industrielle d'un " nouvel appareillage d'écriture et de lecture, non seulement entre êtres humains, mais entre robots et appareils automatiques d'une part, humains d'autre part » (Stiegler, $2013:$ 91).

Pour prendre position dans ce débat, nous avons analysé successivement des corpus d'énoncés en lien avec l'exposition Edward Hopper à Paris 3 et avec le Festival de Cannes 2013 ${ }^{4}$. En nous référant aux cadres de la sociologie de la culture et de la muséologie, nous avons constaté une diversité de situations et de formats d'écritures numériques qui constituent, tant pour le public, les professionnels de la culture que pour les organisateurs de l'événement, le support technologique d'une énonciation renouvelée de l'événement culturel. En travaillant aussi le contenu de ces discours et leur dimension rhétorique (valeurs, idées et représentations), nous constatons que leur réputation de superficialité tient plus à la nature participative du support qu'à la dimension événementielle des deux manifestations.

\section{L'événement Hopper au Grand Palais : le contexte}

12 Avant de proposer l'exposition Hopper, le Grand Palais 5 avait déjà réalisé plusieurs expositions qualifiées par les médias d'" expositions-événements » comme Matisse Picasso (2002-2003) et Monet (2011). Si bien qu'au fil du temps, l'établissement a perfectionné ses stratégies et ses plans de communication jusqu'à l'opération de communication de grande envergure indubitablement réussie pour Edward Hopper, qualifiée par certains journalistes « d'art d'aller chercher le public » (Sophie Cachon, Télérama, le 16 mars 2013). La journaliste égrène les clés de cette réussite : un titre accrocheur simple et facilement interprétable, une héroïsation de l'artiste et du visiteur, un logo 
omniprésent et une campagne de communication divisée en plusieurs temps, qui débute par le «teaser » - ou " publicité énigmatique » - de l'exposition afin de mettre les publics en haleine ; puis l'affichage massif des œuvres les plus connues de l'artiste dans tout Paris, sur les bus, dans le métro, sur Internet et sur les réseaux sociaux ; une politique éditoriale offensive (papier et numérique), des produits dérivés en vente à la boutique du palais et sur l'e-boutique, des partenariats avec les grands médias et les médias spécialisés, un mécénat discrètement mais efficacement valorisé (Starbucks). Bref, une stratégie marketing parfaitement maîtrisée visant à séduire les publics potentiels et à les convaincre que cette exposition est un événement à ne pas manquer sous peine de passer à côté d'une expérience hors normes : "Il y a dix ans, ça n’intéressait personne de faire de la pub sur les bus, mais, depuis, tout le monde a suivi, même quand il n'y a pas matière à événement », raille Patrick Restellini, précurseur du concept d'exposition-événement dans son entretien avec Clio Weickert sur le site de Télérama le 26 mars 2013.

Le Grand Palais publie sur son site internet, durant toute la durée de l'événement, des pages consacrées à l'exposition vedette du moment. Pour Edward Hopper, les internautes qui tapaient le nom du peintre sur leur moteur de recherche habituel se trouvaient instantanément en lien avec la page de l'événement Hopper gérée comme un blog, avec une mise en page et un design qui font coexister les images, les vidéos, les textes, les commentaires des internautes, des entretiens avec le commissaire de l'exposition, des témoignages d'artistes.

14 Le Grand Palais travaille aussi sa présence en réseau, car grâce aux automates numériques, les médias numériques s'apostrophent les uns les autres en permanence, déployant une écriture en perpétuel changement, en permanente recomposition. À partir de notre écran, de notre téléphone ou de notre tablette, il semblerait possible que nous participions ainsi à la transformation de l'institution musée, de ses médiations, mais aussi à la transformation de ses interactions avec le reste du monde. L'internaute-énonciateur collaborerait ainsi, sans toujours s'en rendre compte, avec les opérateurs des télécommunications, aux médiations de l'exposition, à sa sociabilité, à la chaîne des discours et à l'archéologie de sa mémoire discursive (Derrida, 1967).

Selon l'Observatoire des pratiques numériques du Grand Palais, la demande du public numérique pour ce type de dispositif collaboratif serait exponentielle. Cette affirmation validerait de ce fait la conviction de certains experts, selon lesquels l'accomplissement d'une activité culturelle numérique valorisante - utiliser le blog comme prémisse à la visite réelle - va de pair avec un ensemble d'activités et d'échanges sociaux (se renseigner, échanger, suivre les réseaux sociaux, télécharger des reproductions, en parler, lire la critique, attribuer une note ou un jugement, etc.)

\section{S'enquérir des catégories de tweets à propos de l'exposition Edward Hopper}

16 Pour analyser l'écriture numérique de l'événement Festival de Cannes, nous avons ciblé nos investigations sur Twitter, ce qui, nous le verrons plus loin, permet de déceler comment, chaque année, les réseaux sociaux font bouger pour les professionnels, les amateurs et les admirateurs, les frontières imaginaires de la pulsion de voir le Festival à travers le regard des V.I.P., mais aussi les frontières plus objectives de l'espace-t emps de l'événement, bien au-delà de Cannes et de la programmation.

17 Pour Hopper, nous n'avons retenu, parmi les vagues de tweets qui se sont succédé, que ceux qui convergent vers des blogs ou des sites de médias et d'internautes légitimes. C'est la raison pour laquelle nous ne nous attarderons pas sur l'analyse des tweets, énoncés brefs et furtifs dont l'intérêt majeur a été selon nous de tracer une carte de Tendre des visiteurs de l'exposition, scindant en deux groupes distincts les twittos. Ceux qui sont fascinés par l'œuvre du peintre et la médiation du Grand Palais d'une part, ceux qui sont frustrés par le manque d'accessibilité aux œuvres et agacés par le battage publicitaire 
réalisé autour de l'événement d'autre part. De manière très conventionnelle, quantité de tweets se limitent à s'interpeller sur des détails, faire des traits d'humour, "retweeter » sans s'attarder, donner des trucs et astuces - le " gazouillis » évoqué plus loin pour le Festival de Cannes - pour éviter les heures de trop grande affluence, l'attente dans le froid ou sous la pluie.

À la marge, des tweets avisés permettent de converger vers des sites ou des blogs riches en informations, interactions et commentaires critiques de qualité, émanant de professionnels (journalistes, critiques, muséographes, historiens de l'art, artistes) ou d'amateurs (visiteurs éclairés, connaisseurs de l'œuvre d'Hopper, « muséo-geeks »).

\section{Corpus et protocole d'analyse}

Notre protocole d'analyse repose avant tout sur un travail de veille mené sur la Toile dans l'objectif de pouvoir mieux appréhender l'écriture numérique de l'exposition événement Edward Hopper7. À l'issue de cette veille (réalisée pendant la durée de l'exposition), nous avons sélectionné les participations les plus représentatives de la nature et des enjeux des données échangées, soit une trentaine de sites, blogs et articles numériques ainsi qu'une cinquantaine d'échanges sur Twitter évoqués ci-dessus. Certains articles parmi les archives sélectionnées étaient publiés (oral, écrit) sur les sites de radios ou de télévisions (dont France Culture et France Info, TF1, Arte). L'application iPad réalisée par le Grand Palais ${ }^{8}$ faisait également partie de notre corpus de recherche. Faute de place, nous ne l'analysons pas dans cet article car elle forme une médiation autonome et représente par là même un sujet d'analyse sociopragmatique en soi.

\section{Ce que disent les écrits numériques à propos de cette exposition-événement}

L'ensemble des propos et des représentations qui composent les discours conçus par des internautes au cours de l'événement peuvent être regroupés autour de trois thématiques : Hopper, les clichés à propos de l'événement, un regard réflexif ${ }^{9}$ sur ce qu'est une exposition-événement.

21 Premier thème, les discours qui parlent d'Hopper : les gens adorent les expositions monographiques présentant des peintres déjà connus ; Hopper est un artiste qui a conquis le cœur des Français parce qu'il a peint Paris et qu'il a résidé en France, il est normal que l'exposition soit un succès à Paris ; sa peinture donne à voir une Amérique pensive et subtile, loin des clichés et des stéréotypes sur une Amérique pulsionnelle et épaisse ; le commissaire de l'exposition Didier Ottinger valorise l'œuvre d'Hopper en insistant sur les qualités picturales et plastiques des toiles exposées ; la presse - qui était sceptique lors de l'inauguration - a été séduite par l'œuvre, par des tableaux jusque-là inconnus, par l'accrochage et par l'enthousiasme des publics pour Hopper ; les liens étroits évoqués de façon répétitive entre Hopper et le cinéma, notamment les films d'Hitchcock, David Lynch, Wim Wenders ; l'œuvre d'Hopper fait événement car elle permet aux amateurs de remettre en question les dogmes de l'art contemporain mais aussi parce qu'elle donne du plaisir et à penser, à voir autrement notre rapport à l'espace et au temps ; Hopper déconstruit certaines représentations de la femme moderne et de la communication entre homme et femme dans l'univers urbain ; des photographes et des écrivains connus reconnaissent avoir une dette envers son style, son vocabulaire formel, ses thèmes obsessionnels.

22 Deuxième thème, l'exposition Edward Hopper comme média grand public : la comparaison se fait grâce au numérique avec d'autres expositions conçues sur le même format, qui ont remporté des succès grand public (Manet, Monet, Matisse-Picasso...) ; un parcours d'exposition pertinent et facilement compréhensible ; des textes de médiation 
conçus pour les non-experts ; une exposition qui correspond à la demande du public français, un public désormais exigeant et amateur de grandes expositions monographiques ; une exposition qui fait d'Hopper une superstar internationale dont l'œuvre est facilement reconnaissable ; des partenariats avec les grands titres de la presse écrite ; un catalogue de qualité ; des publications numériques accessibles ; la prolongation de l'exposition pour permettre à plus de monde de s'y rendre, avec des tarifs et des horaires exceptionnellement alléchants ; un commissaire d'exposition particulièrement présent dans les médias et capable d'avoir fait déplacer des œuvres jusque-là jamais vues en France ; une exposition qui met en avant les influences d'Hopper sur d'autres artistes ; la mise en évidence du fait que le langage des experts donne à voir autrement la peinture ; aller voir Edward Hopper est un « devoir imposé » par l'opinion publique ainsi relayée sur Internet et les médias.

Troisième thème, un regard réflexif porté sur l'événement-exposition et sur la participation à cet événement : ce sont les médias qui, surexposant sans trêve l'expositionévénement, en font un événement médiatique ; le propre de l'événement est de se dérouler en même temps sous les yeux de ceux qui le vivent et sous le regard des médias qui le rapportent ; l'événement est avant tout une affaire de spécialistes de la communication et d'experts qui travaillent l'image d'un artiste comme celle d'un produit de luxe ; il y a événement parce qu'il y a, autour de l'exposition, la création d'une somme d'événements connexes (publications, jeux, prolongation, invitations, conférences, films, produits dérivés, etc.) ; certains intellectuels (par exemple Antoine Compagnon) se disent honteux d'avoir suivi le mouvement, ironisent sur les ficelles marketing, mais avouent avoir suivi l'injonction à se rendre au Grand Palais et en profitent pour écrire une critique d'Hopper à leur manière ; les expositions-événements sont soutenues par le ministère de la Culture qui les considère comme des propulseurs pour augmenter le nombre de visiteurs dans les institutions muséales et patrimoniales ; l'exposition-événement permet aux internautes d'échanger entre eux, de se renvoyer une image positive, de parler d'eux-mêmes de manière flatteuse.

\section{Des jeux de langage confirment la mécanique du copier-coller du numérique}

$24 \quad$ Redites, reprises, formules toutes faites, figures de style éculées, humour plus ou moins décalé, les phrases et les énoncés des internautes et des journalistes ne manquent pas de stéréotypes langagiers qui confinent aux jeux de langage pour se comprendre entre soi sans même avoir à développer sa pensée (Wittgenstein, 1961). Ces registres sémiotiques qui se rapprochent souvent du cliché peuvent être regroupés en deux catégories : les informations pratiques et la critique, plutôt ironique, de l'événement.

25 Informations pratiques : les sites touristiques mais aussi la plupart des blogs et articles fournissent des informations pratiques sur le lieu, les horaires, les moyens de se rendre au Grand Palais ; les astuces pour éviter les longues files d'attente dans le froid ; les trucs pour bénéficier de tarifs préférentiels ; tous sont écrits avec une prédilection pour un vocabulaire expert mais pas abscons ; le thème de la visite d'exposition marathon revient régulièrement - difficultés, épuisement, quête récompensée, plaisir final assuré.

Description de l'exposition et ton ironique à propos de l'événement : on fournit des chiffres, des chiffres et encore des chiffres pour confirmer la force d'attraction de l'événement sur le grand public ; nombreux sont les propos qui ironisent et critiquent ce qu'ils considèrent comme une résignation des mondes de l'art à la consommation de masse ; récurrence des souvenirs en lien avec d'autres expositions blockbusters décevantes auxquelles on a participé ; la presse ne s'est intéressée à l'exposition qu'après que le grand public l'eut plébiscitée en venant massivement au Grand palais et en investissant les réseaux sociaux ; les champs lexicaux récurrents dans la description des œuvres sont ceux de l'étrangeté, de la mélancolie, de l'Amérique, de la difficulté à communiquer ; les 
visiteurs sont décrits comme des héros capables d'endurer le froid et la fatigue pour accéder au Saint Graal ; les témoignages de visiteurs connus sont repris à l'envi pour valider le caractère exceptionnel de l'événement ; très peu de connaissances sérieuses sur le peintre et son œuvre ; on cherche à rassurer ses lecteurs en précisant qu'on verra du déjà connu, du facile à voir ; on joue sur les émotions ressenties devant les œuvres ; « une œuvre vue, c'est une œuvre faite »; « il faut y aller pour dire qu'on y est allé ».

\section{Que retiendra-t-on de cette exposition ouverte au public pendant cinq mois à la fin de l'année 2012 ?}

La mémoire de cette exposition-événement sur le Web 2.0 : on vient de le voir, ce qu’il a été convenu de qualifier de " l'événement Hopper au Grand Palais » a donné lieu sur la Toile à des foisonnements de points de vue, de réactions, d'analyses, de formes de réceptions extrêmement variées tant sur le plan des sémiotiques d'écriture que des contenus élaborés, échangés, repris et transformés par les modalités de production et de transmission de l'écriture numérique. On ne mesure certainement pas encore à quel point ces modalités, qui relèvent autant de la technologie que des usages, façonnent déjà la mémoire que nous aurons demain des relations que nous instaurons aujourd'hui entre nos rapports collectifs et intimes à l'art et à nos pratiques culturelles. Quel sera le droit à l'oubli de cet événement culturel dès février 2013, alors que, déjà, Dynamo, l'autre exposition-événement du Grand Palais pour l'année 2013, lance son teaser dans le métro parisien?

Les traces sur le Web permettront de se souvenir qu'Edward Hopper faisait partie à l'époque des six expositions les plus visitées depuis 1967 - 800 ooo entrées en trois mois, dont près de 50 ooo lors des deux jours de prolongation -, deuxième record de fréquentation après le succès de Monet en 2011 - 910 ooo visiteurs. Le nom du commissaire, Didier Ottinger, restera synonyme de professionnalisme, tant pour le montage de l'exposition - prêt d'œuvres rares du monde entier -, que pour la qualité de ses écrits scientifiques sur Hopper (catalogue et applications pour tablettes ou téléphone mobile, C.D.ROM), et pour sa compétence à mettre en résonance l'œuvre d'Hopper avec des cinéastes, des photographes, des écrivains et des auteurs dramatiques. On saura que le Grand Palais et son service des publics et des manifestations événementielles ont fait preuve alors d'un plan de communication offensif et jusqu'alors inégalé en France pour aller chercher les visiteurs, les faire venir, adapter les stratégies du marketing des produits de luxe à un artiste peintre américain jusque-là peu connu du grand public, mais dont l'œuvre figurative est capable de faire sens pour les visiteurs français comme pour les touristes étrangers. On se rappellera que les internautes ont été très nombreux à participer aux interactions entre visiteurs effectifs, visiteurs potentiels, entre journalistes et professionnels de la culture, entre gens des musées et béotiens des beaux-arts, entre l'institution et ses publics numériques. On pourra également, grâce à toutes les reproductions des œuvres, des vidéos de l'exposition et du Grand Palais, grâce aussi aux bandes-son et aux photos numériques de visiteurs photographes amateurs, se représenter l'œuvre d'Hopper et l'événement auquel cette exposition donne lieu.

\section{Le festival de Cannes en régime d'attestations numériques de présence spectatorielle}


Le Festival de Cannes, pour sa part, fonctionne sur un registre différent où se télescopent quotidiennement durant près d'une dizaine de jours « un » public et un seul, marquant sa présence sur une durée allant d'un jour à la totalité de l'événement, un événement dont le corps est constitué d'une programmation de films inédits proposés par l'institution, qui met l'auteur au centre. Le principe de l'événement est qu'on ne peut pas tout voir, ce qui implique une masse d'information considérable et signifie également que se crée plus ou moins consciemment une volonté de trouver " the place to be » pour participer au mieux à la manifestation. Cette place n'existe cependant pas puisque chacun devient témoin privilégié. Twitter va donc permettre à tout un chacun de se doter d'une ubiquité numérique pour renforcer sa conscience de l'événement puisqu'il est impossible d'assister à tout en même temps. Le réseau social va, de fait, transformer ses publics en témoins assistés et doter la direction du Festival d'un outil de légitimation de ce qu'est la réalité de ce qu'elle voudrait partager réellement de l'événement. Lorsqu'on a la curiosité de consulter le règlement qui régit cette association d'intérêt public qu'est le Festival de Cannes, on découvre en exergue une phrase définitoire de la manifestation signée de Jean Cocteau qui en fut le président en 1953 et 1954 : " Le Festival est un no man's land apolitique, un microcosme de ce que serait le monde si les hommes pouvaient prendre des contacts directs et parler la même langue. » Bien sûr, il ne s'agit pas de lire ici la phrase du poète comme une sorte de prémonition fulgurante de ce que l'on appelle aujourd'hui « les réseaux sociaux ", mais plutôt d'imaginer comment lesdits réseaux sociaux ont trouvé aujourd'hui une place quasi évidente d'où se réifie la dynamique même des régimes de participation à certains événements culturels en offrant une possibilité d'expression inédite et immédiate à leurs participants. À Cannes, si l'on distingue toujours assez aisément ceux qui appartiennent à l'organisation du Festival, les invités, les compétiteurs, les festivaliers, les professionnels du marché, les journalistes, les critiques et les badauds, les réseaux sociaux s'imposent comme une nouvelle incitation sociale pour exhiber le fait qu'on est « de » l'événement, tout en assignant à ceux qui entrent dans leur jeu une place inédite de témoins de la manifestation en les autorisant à consigner à leur manière cette dernière. Pas n'importe quels témoins. Ce sont des témoins assistés au sens où Twitter leur confère une place plus ou moins légitime en fonction de ce qu'ils écrivent, de ce qu'ils décrivent, de ceux qui les suivent et de ceux, qu'eux-mêmes, ils suivent. Ainsi les registres numériques de participation au Festival de Cannes définissent-ils, à proprement parler, les conditions sociales d'une attestation personnelle d'association et de coopération à l'événement.

Désormais, ce qu'on découvre dans un réseau social comme Twitter qui, plus que dans n'importe quel autre réseau, fonctionne majoritairement sur le témoignage de l'instant soit en 140 signes soit via une image légendée ou non, ce n'est plus une version unique et unifiée du Festival, mais plusieurs dizaines. Et quand plus de dix versions font " diversion », la version officielle - reconnaissable à ce qu'elle se drape de la tautologie de l'officialité institutionnelle - tente à nouveau de reprendre sa place dans le réseau pour réguler, réorienter, redresser les " attentions obliques » des twittos cannois, festivaliers usagers de Twitter-. On ne comprend jamais si bien l'idée d'attention oblique qu'en regardant les films de Jacques Tati. La caméra y semble toujours en quête de multiples narrations parallèles à ce qui, pour la plupart d'entre nous, constituerait « naturellement » le cœur de l'histoire. Pour le réalisateur de Playtime, les grincements de portes dans des immeubles high-tech, les ombres portées sur des surfaces impromptues, les forces déployées par tout un chacun pour maintenir l'ordre apparent des choses sont autant de fausses évidences qui façonnent les décors dans lesquels nous évoluons dans la plus parfaite des insouciances et constituent un système de valeurs à part entière, un regard que les critères classiques de la consommation culturelle considèrent volontiers comme un regard distrait. Ce regard distrait, cette attention oblique, décrit avec une très grande justesse par le sociologue Richard Hoggart, est sans doute l'une des voies les plus sûres pour accrocher toutes ces interprétations nonchalantes souvent éloignées de la vision monolithique du monde telle que les cultures dominantes ou « officielles » tentent de l'éla- 
borer et de l'imposer. Ces interprétations nonchalantes, elles, " en prennent et en laissent » pour reconstruire une autre version de l'histoire tout aussi cohérente, mais faite de distance et de méfiance, et d'où l'on appréhende parfois mieux la place et le rôle de ce que l'on voudrait nous faire prendre tantôt pour le centre du monde social, tantôt pour ses périphéries.

31 En tant qu'événement institutionnel, le Festival de Cannes et, plus exactement, les images et les signes médiatiques qui tentent d'instruire une vision univoque et contrôlée de l'événement, travaillent précisément à forcer l'attention de tous les participants sur quelques points de fixation de la manifestation et tolèrent mal les attentions obliques. Au demeurant, il réside dans la force centrifuge des images médiatiques du Festival un joli paradoxe qui fonde la frénésie du regard participant à Cannes. Ainsi, les vitres teintées des limousines de luxe, surtout lorsqu'elles sont fermées, rappellent à tous ici, et ce, dans une démesure sans relâche, qu'il y aurait peut-être toujours mieux à voir que ce que le dispositif festivalier nous presse de regarder. Voir, voir mieux, voir pire, voir plus, voir plus ou moins, voir plus vite, voir le visible et l'invisible, voir au travers ou au détour, voir ce qu'il ne faut pas voir, voir comment les autres voient, se voir, revoir, se revoir, ne pas avoir pu voir, authenticité du voir, percevoir cette vérité aveuglante du voir : le Festival reconstruit chaque année une véritable écologie du voir saturée de symboles qui recouvrent une grande partie de la ville et qui semblent y délimiter les frontières, mais aussi le temps de l'action, c'est-à-dire le temps du Festival.

32 Tout comme l'exposition-événement Edward Hopper, Cannes établit un véritable dispositif d'accès au visible et au non-visible. Ce visible se laisse même dépeindre comme une véritable économie de l'apparition par laquelle Cannes s'institue comme spectacle aux yeux de tous ses participants (Claverie, 2001). De fait, il y a ceux qui voient, ceux qui ne voient pas, ceux qui ne font qu'entrevoir ou apercevoir. En 1955, le sociologue Edgar Morin remarque déjà que " la question que l'on pose à celui qui rentre de Cannes est d'abord "Quelles vedettes avez-vous vues" et ensuite "Quels films" [...]. Puis il doit répondre à la deuxième question, la question clé, celle qui implique et explique toute la mythologie du festival "Est-elle aussi bien qu'à l'écran, aussi jolie, aussi fraîche", etc. Car le vrai problème est celui de la confrontation du mythe et de la réalité, des apparences et de l'essence ». Pour les Cannois « d'origine », les touristes et les badauds qui fournissent l'essentiel des figurants peuplant les abords du palais, la manifestation est circonscrite à quelques espaces d'extérieur, symboliques, hautement médiatisés et puissamment investis. Ceux qui sont accrédités par le Festival de Cannes ou le Marché du Film professionnels du cinéma, institutions, médias et spectateurs spécialisés ou cinéphiles partagent, eux, avec les organisateurs, les producteurs et les artistes du « premier cercle » le privilège d'un accès direct - sporadique ou continu - aux offres du Festival et aux soirées privées. Si un réseau social comme Twitter prend tout son sens dans l'événement, c'est qu'il donne l'apparence de structurer une sorte de communauté numérique entre le cercle le plus proche de l'événement en train de se faire au cœur du palais, d'un palace ou d'une soirée, et le cercle plus éloigné constitué de ceux qui sont présents mais qui - parce qu'ils n'ont pas le « ticket » - ne peuvent assister à l'événement : être à quelques mètres de là où les choses semblent se passer, savoir qu'elles ont lieu dans un même espace-temps, traquer une information attestée qui donne corps et substance à ces choses définissent en soi une modalité de participation via les réseaux sociaux qui servent aussi - bien évidemment - à relier ceux qui sont encore plus éloignés de l'événement, n'étant pas géographiquement présents à Cannes, et qui suivent le fil des aventures des twittos participants qu'ils connaissent. Aussi, comprendre les conditions sociales d'une attestation personnelle d'association et de coopération à un événement tel que le Festival de Cannes via Twitter consiste à dépasser les apparences entretenues d'une communauté numérique fédérée autour de l'événement ${ }^{10}$ et à tenter de répondre à trois questions susceptibles de poser les fondements d'une sociologie critique de ce que l'on produit sur un réseau social donné durant un événement culturel donné : 
1. Qui écrit des tweets et pourquoi ?

2. Qui lit ces tweets produits, comment et pourquoi les lit-on?

3. Comment évalue-t-on ces tweets et pourquoi ?

(1) Écrire sur Twitter suppose que l'on soit inscrit sur le réseau social et que l'on se soit saisi des rudiments d'écriture et des codes minimaux qui permettent de produire une information sur ledit réseau. Les 140 signes autorisés dans un tweet ou la photo légendée instruisent un format contraint qui centre le contenu des messages sur la divulgation d'un état d'âme, d'une citation écrite ou visuelle, de la mise en place d'une conversation s'apparentant à l'échange public de SMS, et, en l'occurrence, lorsqu'on participe à un événement, d'une volonté de témoigner de ce que l'on voit et que l'on souhaite faire partager.

(2) Ceux qui lisent les tweets produits sont les "followers ». Ils " suivent » un producteur de tweets - individu ou institution - et veulent faire lien avec ce dernier parce qu'ils estiment que l'information qu'il leur apporte est légitime. Ils sont abonnés aux comptes de ceux qu'ils choisissent de lire et avec lesquels ils aspirent à entretenir un lien privilégié. À Cannes par exemple, on observe des twittos qui suivent en direct « leur » star quand celle-ci est sur Twitter, qu'elle propose sa vision du Festival ou informe, plus simplement, de là où elle se trouve à un moment donné de la manifestation et de ce qu'elle y fait. La star s'abonne rarement aux comptes de ses followers - si c'est le cas, c'est d'ailleurs une consécration pour le follower qui devient alors "following », " suivi par sa star ». Elle est généralement abonnée à ceux qui sont déjà ses amis dans la « vraie »vie, rendant, par le fait, publiques ses connivences amicalo-numériques. Il est courant que les followers d'une star retweetent les tweets de leur star. ; ce qui génère un effet gigogne des messages déposés par celle-ci sur Twitter. L'exemple du starfan est instructif car il permet de comprendre assez simplement le mécanisme et l'intérêt du réseau social. L’intérêt du tweet à

Cannes tient à la force testimoniale dont il est porteur, une force qui dépend de la substance du message et de sa proximité spatiotemporelle avec le lecteur-follower. Un bon tweet contient une information qui pourra être, au sens le plus terre-à-terre, réexploitée par le follower car elle vient alimenter ses intérêts immédiats dans le cadre de la manifestation cannoise : où se passe-t-il quelque chose maintenant?

(3) L'évaluation d'un tweet à Cannes tient à la pertinence du message partagé, une pertinence assertorique qui relève de son intérêt tantôt prescriptif-informatif - quel film voir ? Où se trouve telle fête et comment y entrer ? -, tantôt de partage d'avis, de citations, d'états d'âme ou d'opinions, un intérêt qui se mesure donc à l'aune de l'événement et des faits festivaliers qu'il génère afin d'enrichir le sens même de chaque contribution personnelle au sein du collectif participant. Il faut noter que le fait qu'à Cannes, outre l'existence du compte officiel du Festival, les plus hauts responsables de la manifestation le président Gilles Jacob et le délégué artistique Thierry Frémaux ${ }^{11}$ - aient fait le choix d'ouvrir leur compte personnel sur Twitter renforce la dynamique participative à la manifestation et, en conséquence, entretient l'illusion d'une communauté « cinéphiliconnectée » organisée autour de l'événement.

\section{Des tweets et « des » Cannes}

Là où J. Cocteau parle de "no man's land apolitique où l'on peut prendre des contacts directs et parler la même langue », l'anthropologue Marc Augé, pour sa part, développe le concept sociologique de "non-l ieu », un espace non marqué par l'attachement historique et culturel d'individus ou de groupes audit espace, mais répondant plutôt à un principe de contractualité solitaire. « [Dans] les non-lieux réels de la surmodernité, [...] les individus sont censés n'interagir qu'avec des textes sans autres énonciateurs que des personnes "morales" ou des institutions - aéroports, compagnies d'aviation, ministère des 
Transports, sociétés commerciales, police de la route, municipalités - dont la présence se devine [.] derrière les [.] innombrables "supports" (panneaux, écrans, affiches) qui font partie intégrante du paysage contemporain. » (Augé, 1992.) Les réseaux sociaux, parce qu'ils distillent leurs messages dans un espace-temps en créant un double virtuel des personnes morales, des institutions mais également de personnes réelles, offrent la sensation d'énonciateurs plus vivants, même lorsque le doute sur celui ou celle qui poste réellement les messages subsiste dans nombre de situations (Jarassé, 2013).

Durant le Festival 2012, le délégué artistique de Cannes T. Frémaux publiait entre 9 et 20 tweets par jour sur son compte « officiel mais personnel12 ${ }^{12}$ - peu de doute ici donc sur le fait qu'il soit bien l'auteur de ses propres messages. Durant le Festival 2013, réduction considérable, il publie entre o et 5 tweets par jour. Les assertions de T. Frémaux sont habitées par la fonction institutionnelle qu'il occupe, dévoilant parfois sa conception de ladite fonction grâce à une citation - « Je m'en sortirai toujours, seul, par le travail (génial et regretté Philippe Khorsand) " - ou à une assertion à portée existentielle ou pragmatique : "C'est parce qu'on s'intéresse à ce qui ne nous ressemble pas qu'on aime (tous) le cinéma. Et on ne privilégie rien du tout. » "Vous savez quoi ? À Cannes comme ailleurs, les jurés votent selon leurs... convictions. Décevant, non ? Rassurant, plutôt, hein. » Plus souvent, le délégué général du Festival exprime son agacement face aux rumeurs qui corsètent la manifestation : "Quand on colporte les rumeurs en les (re)tweetant on prend le risque d'avoir l'air bête si elles sont fausses. " « Il y a beaucoup de bêtises, d'erreurs et de clichés 100 fois répétés mais voilà qui dément l'accueil unanime fait à Cannes 2013. "

C'est sans doute cette tension permanente entre le portage officiel, nécessaire, de l'institution et la manière dont la circulation des on-dit ébranle l'éthique du fonctionnement attendu du Festival qui conduit T. Frémaux à écrire : « Il n'en a jamais été question. Cessons de s'obliger à démentir les rumeurs. " En s'obligeant lui-même d'une année sur l'autre à d'une part ne plus répondre aux racontars, et d'autre part à prendre ses distances avec le réseau social en énonçant incidemment ce que devrait être, selon lui, l'usage moral de Twitter à Cannes, c'est-à-dire ce qu'on peut ou ne pas faire en termes de messages : «C'est une plaisanterie pour la salle, pas pour que ça soit bêtement repris par Twitter. On va finir par ne plus rien dire. » " La sélection n'est pas notre vision du monde mais celle des cinéastes. Et il faut avoir vu tous les films si on veut (1/2). faire des analyses ou des conclusions définitives ou des tweets de 100 caractères (2/2). »

Depuis qu'il n'est plus délégué artistique mais président du Festival de Cannes, G. Jacob jouit, pour sa part, pleinement d'un statut prestigieux qui le place en surplomb de la manifestation en lui consacrant une place de témoin privilégié. On a vu ainsi, voici quelques années, G. Jacob se munir d'un petit appareil photo numérique et, posté en haut des marches depuis la place qui est la sienne, capturer des moments qu'aucun photographe ou journaliste accrédité ne pourrait saisir mieux que lui. G. Jacob cultive sa place de témoin unique et lorsqu'il ouvre son compte Twitter, il va asservir celui-ci pour en faire un outil léger du témoignage direct et immédiat prolongeant les gestes et les regards qu'il avait initiés avec ses « simples » prises de vue. Le président G. Jacob est un twitto qui a trouvé très rapidement son " régime de croisière ", publiant autour de 12 tweets par jour de Festival. Suivi par environ $15 \%$ de followers de plus que T. Frémaux pour une production de $31 \%$ de tweets de plus que le délégué artistique actuel, et bien qu'il soit président - c'est-à-dire plus haut dans la hiérarchie institutionnelle de Cannes -, G. Jacob apparaît plus en prise avec les intérêts des festivaliers cinéphiles, là où $\mathrm{T}$. Frémaux est en dialogue plus direct avec les professionnels et les médias. De fait, les messages de G. Jacob se déploient avec une écriture numérique stabilisée par une volonté maîtrisée du tweet qui vise à faire partager une vision idéalisée du monde cannois : «Un palais où on peut se perdre et y finir ses jours », " J'ai prévenu le grand producteur anglais Jeremy Thomas que j'allais tweeter sa montre », "Un bon jury est un jury qui, à la fin, après quinze jours de vie commune, de débats, de partage, jure de se revoir - et tient promesse », « La ministre Aurélie Filippetti retrace, pour la profession réunie, les enjeux 
du combat pour l'exception culturelle ». Mis bout à bout, les tweets de G. Jacob, dénués de fautes d'orthographe, de fautes de ponctuation ou de cadrages incertains semblent vouloir " faire histoire ». On conçoit que son plus grand plaisir relève de la photo rare tweetée et légendée d'un texte drôle, anecdotique ou poétique. Twitter devient dès lors, dans ce type de situation, «bien plus que du témoignage, [...] un lieu d'échange, de partage [irréductible] à une somme de gazouillis. [On est ici loin] du fantasme de l'information qui pourrait être faite par tous, tout le temps, à propos de n'importe quel sujet. Or l'information c'est d'abord du traitement, de la hiérarchisation » (Spies, 2011).

Il n'en demeure pas moins qu'à Cannes, pour reprendre les mots de M. Augé (1992), "l'individu se veut un monde. Il entend interpréter par et pour lui-même les informations qui lui sont délivrées ». De fait, l'usage des réseaux sociaux renforce cette individualisation des démarches et les effets de reproduction et de stéréotypie qui échappent en totalité ou en partie à la conscience des festivaliers. L'espace cannois offre en lui-même un non-l ieu " principe de sens » pour ceux qui le colonisent le temps de la manifestation cinématographique. De plus, il fournit un " principe d’intelligibilité » pour les lecteurs des tweets cannois, tweets qui puisent leur valeur tant de la légitimité tirée de la place de témoin occupée par celui qui les poste que de l'esthétique globale du compte de ce dernier. Le festivalier-witto, qui n'utilise pas Twitter comme moyen d'autopromotion parce qu'il est accrédité en tant que journaliste - nombre de journalistes signalent grâce à Twitter qu'un de leurs reportages est lisible, visible ou écoutable dans son média d'appartenance ou comme professionnel en charge de vendre un film, se retrouve le plus souvent sur le réseau pour exprimer sa relation à l'univers cannois et principalement aux œuvres cinématographiques. Cette relation traduit une posture qui implique spécifiquement son identité personnelle dans le moment cinématographique, c'est-à-dire avant, pendant et après le film : "Sortie de Gatsby. Je croise Léo dans l'escalier. Il me regarde », " $\mathrm{Au}$ cinquième rang à l'orchestre, à côté d'un visage qui me dit quelque chose. Qui m'aide ? », " Ai marché sur la robe de Julie Delpy. L'année dernière, c'était Gong Lee », « Dix heures en salles aujourd'hui. La moitié à dormir ", "Fête Honoré. Chiara et Garrel chantent devant Deneuve. T'es juste derrière, touche sa main ». Twitter permet à ceux qui ont pris véritablement conscience qu'ils ont de fortes chances d'être lus de restaurer une fonction sociale primaire de l'expérience esthétique car elle fait sortir la relation du public à l'objet cinématographique pour s'ouvrir " au niveau de l'identification esthétique spontanée qui touche, bouleverse, qui fait admirer, pleurer ou rire par sympathie, et que seul le snobisme peut considérer comme vulgaire » (Jauss, 1978). D'un message à l'autre, on peut constater que les écarts sont encore très grands au regard de leur potentiel de " dire le monde situé » de l'événement festivalier. Il est sans doute trop tôt pour prendre une mesure effective de ces formes d'écritures de témoignage car on remarque combien elles évoluent d'abord lorsqu'elles se confrontent à l'altérité de ceux qui partagent la même pratique numérique. L'accomplissement du sens de ces écritures se fera, comme toutes les écritures de témoignage qui ont précédé Twitter, en " extériorité ». Car les témoignages partagés du petit peuple des festivaliers cannois fonctionnent bel et bien en s'interpellant les uns les autres, animés d'une volonté de construire une fonction de liaison du langage qui rappelle la responsabilité de chacun dans l'usage qui est fait du réseau social et ce, en confirmant régulièrement les principes intuitifs de ce qui devrait être constitutif d'une communauté de semblables. Le temps d'un événement, ces nouvelles pratiques numériques du témoignage écrit aspirent à créer une véritable solidarité communicationnelle. Et, comme le précise Renaud Dulong à propos de l'activité de témoin oculaire, il s'agit bien là de « raconter la vérité telle qu'elle peut être transmise d’homme à homme » (Dulong, 1984).

\section{Conclusion}

Qu'est-ce qui s'écrit dans les écritures numériques qui collaborent à des événements culturels comme le Festival de Cannes ou l'exposition Edward Hopper à Paris ? C'est la 
question que nous avons posée en prenant comme objet d'analyse ces deux terrains artistiques ainsi qu'un corpus constitué notamment de tweets pour le Festival de Cannes et de blogs pour l'exposition du Grand Palais. À bien des égards, ces écritures numériques confirment que des événements culturels aussi prestigieux attestent d'une envie aiguisée d'association et de coopération à l'événement. Visiteurs, festivaliers, badauds, professionnels, critiques et journalistes inscrivent leurs didascalies à l'intention de leurs pairs potentiels sur le Web et sur les réseaux sociaux. Chacun cherche à donner sa vision de l'événement, son attestation oblique, écrivant ainsi des pages culturelles à sa manière, jonglant avec ses formules, ses mots d'esprit et ses langages. Nous avons par ailleurs à plusieurs moments insisté sur la puissance normative des robots (format twitter, design graphique automatisé des plateformes de blogs) qui mettent en forme ces interactions sociales en leur donnant l'apparence trompeuse d'échanges épistolaires spontanés, de microrécits sincères. Nous avons également mis en évidence des phénomènes singuliers de reprise en main de la version officielle de l'événement culturel : recours à la technique du discours indirect qui puise dans les propos du conservateur du musée d'Art moderne de la Ville de Paris, commissaire de l'exposition Edward Hopper ; et stratégie de recouvrement du bavardage des amateurs par les rares - et donc précieux - tweets du délégué artistique et du président du Festival de Cannes. Bref, nous avons mis en évidence ce qui caractérise selon nous l'écriture numérique de l'événement culturel : cette tension constante entre le goût des amateurs à se dire en s'écrivant pendant l'événement et la prégnance permanente de la coercition mathématique de l'informatique sur les écritures participatives numériques.

Pour conclure, nous cédons d'abord la parole à un écrivain malicieux et à un théoricien érudit. Car, parce qu'ils sont circonscrits dans l'espace-temps défini de l'événement culturel, les écrits numériques accompagnant ces manifestations répondent bien aux attendus de ce qui fait la force de la littérature moderne qui, comme le pensait Italo Calvino, tient dans sa volonté de donner la parole à tout ce qui est resté non exprimé (Calvino, 1984). La participation aux écritures numériques d'un événement culturel, dès lors qu'elle relève d'une responsabilité personnalisée d'un acteur, vient charger l'événement de nouveaux savoirs, de valeurs morales, d'expériences pour métamorphoser l'événement à la lumière de faisceaux de témoignages contrebalançant parfois la version institutionnelle et officielle qu'on aimerait en donner. C'est ce phénomène qu'Yves Jeanneret appelle « trivialité », terme entendu au sens où les actes de communication - on tend à négliger trop souvent cette évidence que révèlent les réseaux sociaux en situation événementielle - sont nécessaires à l'appropriation et à l'existence sociale même des êtres culturels (Jeanneret, 2008) et aux modalités par lesquelles s'inventent et se réinventent nos formes de transmission (Malinas, 2008).

Les deux événements culturels considérés dans cet article sont dotés d'une légitimité artistique et économique patente, que confirme en quelque sorte l'écume des échanges numériques constatée à leur propos. Peut-on pour autant généraliser ce phénomène de trivialité communicationnelle à des événements culturels originaux, ou à des formes culturelles mineures ou modestes qui n'intéressent ni les fournisseurs d'accès ni les géants de l'économie numérique ? Sinon, devrait-on en conclure que le numérique " profiterait surtout aux riches "? La réponse à ce questionnement ne se limitera pas à une tension binaire entre le politique et l'économique à propos des événements culturels ; l'avenir du lien entre culture et numérique dépendra avant tout de notre faculté collective à défendre le sensible et l'imagination, autrement dit, à ne pas laisser les algorithmes écrire à notre place.

\section{Bibliographie}

Andreacola (Florence), Poli (Marie-Sylvie) \& Sanjuan (Éric). 2013. " Musée et numérique : Quelle vision du participatif ? » in Actes du Colloque scientifique Ludovia 2013 - 26-29 août 2013. Salvagnac : Association Culture numérique. 
Augé (Marc). 1992. Non-Lieux. Introduction à une anthropologie de la surmodernité. Paris : Seuil, coll. « Librairie du XXIe siècle ».

Becker (Howard). 1988. Les Mondes de l'art. Paris : Flammarion, coll. " Champs Arts ».

Benhamou (Françoise). 2012. Économie du patrimoine culturel. Paris : La Découverte, coll. « Repères ».

Bourdieu (Pierre). 1979. La Distinction. Critique sociale du jugement. Paris : Les Éditions de Minuit, coll. « Le sens commun ».

Brockmeier (Jens). 2012. «Écriture et mémoire », p. 201-216 in Écritures : Sur les traces de Jack Goody/ sous la direction d'Éric Guichard. Lyon : Presses de l'Enssib, coll. « Papiers ».

Calvino (Italo). 1984. La Machine Littérature. Paris : Seuil.

Caron (Daniel-J.). 2011. Web HT.o. Pour une société informée : La pertinence numérique et ses défis pour les sociétés démocratiques au XXIe siècle. Paris : Éditions Hermann, coll. « Cultures numériques ».

Champagne (Patrick). 2000. «L'événement comme enjeu ». Réseaux, 100, p. 403-426.

Chantepie (Philippe) \& Le Diberder (Alain). 2010. Révolution numérique et industries culturelles. Paris : La Découverte, coll. « Repères ».

Chardel (Pierre-Antoine) \& Rockhill (Gabriel) (sous la dir. de). 2009. Technologies de contrôle dans la mondialisation : Enjeux éthiques, politiques et esthétiques. Paris : Éditions Kimé, coll. «Philosophie en cours ».

Clair (Jean). 2007. Malaise dans les musées. Paris : Flammarion, coll. « Café Voltaire ».

Claverie (Élisabeth). 2001. "Cannes et chicanes : "Voir à Cannes ?” », p. 53-63 in Aux Marches du palais. Le Festival de Cannes sous le regard des sciences sociales/sous la direction d'Emmanuel Ethis. Paris : La Documentation française.

Derrida (Jacques). 1967. L'Écriture et la Différence. Paris : Seuil.

Doueihi (Milad). 2011. Pour un humanisme numérique. Paris : Seuil, coll. « Librairie du XXIe siècle ».

Dulong (Renaud). 1998. Le Témoin oculaire, les conditions sociales de l'attestation personnelle. Paris : Éditions de l'École des hautes études en sciences sociales, coll. « Recherches d'histoire et de sciences sociales ».

Eidelman (Jacqueline). 2010. « Du nonpublic des musées au public de la gratuité ». Loisirs et société, 32, p. 174-201. Presses de l'université du Québec.

Ethis (Emmanuel). 2014. Sociologie du cinéma et de ses publics. Paris : Armand Colin, coll. « Sociologie128».

Gaillard (Julien), El Beze (Marc) \& Ethis (Emmanuel). 2013. « Flash Reactivity : Adaptative Model in Recommender Systems » in Actes du World Congress in Computer Science, Computer Engineering and Applied Computing, 22-25 juillet 2013. Las Vegas (USA), Worldcomp.

Gaudreault (André) \& Jost (André) (dir.). 2000. " Présentation de "À la croisée des médias" ». Sociétés et Représentations, 9, p. 5-8.

Goody (Jack). 1994. Entre l'oralité et l'écriture. Paris : Presses universitaires de France, coll. « Ethnologies ».

Hall (Stuart). 2007. Identités et cultures. Politiques des Cultural Studies. Paris : Éditions Amsterdam.

Jacobi (Daniel). 2013. « Exposition temporaire et accélération : La fin d'un paradigme ? ». La Lettre de l'OCIM, 150, p. 15-24.

Jarrassé (Jim) 2013, "Qui se cache derrière les comptes Twitter des ministres ? ». Le Figaro, 6 août 2013.

Jauss (Hans Robert). 1978. Pour une esthétique de la réception. Paris : Gallimard.

Jeanneret (Yves) \& Tardy (Cécile) (dir.). 2007. L’Écriture des médias informatisés : Espaces de pratiques. Paris : Hermès Science-Lavoisier, coll. "Systèmes d'information et organisations documentaires ».

Jeanneret (Yves). 2008. Penser la trivialité : Volume 1 : La vie triviale des êtres culturels. Paris : Hermès Science-Lavoisier, coll. « Communication, médiation et construits sociaux ».

Jouët (Josiane). 2001. " Des usages de la télématique aux Internet Studies », p. 45-90 in Communiquer à l'ère du numérique/sous la direction de Julie Denouël \& Fabien Granjon. Paris : Presses des Mines, coll. « Sciences sociales ».

Jutand (Francis). (dir.). 2013. "Les métamorphoses de l'espace public à l'ère numérique », p. 15-32 in La Métamorphose numérique : Vers une société de la connaissance et de la coopération. Paris : 
Alternatives, coll. « Manifesto ».

Licoppe (Christian) \& Dreatta (Laura). 2013. « Des smart grids aux quantified self, technologies réflexives et gouvernement par les traces », p. 66-72 in La Métamorphose numérique : Vers une société de la connaissance et de la coopération. Paris : Alternatives, coll. " Manifesto ».

Malinas (Damien). 2008. Portrait des festivaliers d'Avignon. Transmettre une fois ? Pour toujours ? Grenoble : Presses universitaires de Grenoble, coll. « Art, culture, publics ».

Merzeau (Louise). 2013. «Éditorialisation collaborative d'un événement : L'exemple des Entretiens du nouveau monde industriel 2012 ». Communication et Organisation, 43, p. 105-122.

Morin (Edgar). 1955. « Notes pour une sociologie du Festival de Cannes ». Les Temps Modernes, 114. Paris : Gallimard.

Nora (Pierre). 1972. « L'événement monstre ». Communications, 18, p. 162-172.

DOI : $10.3406 /$ comm.1972.1272

Paveau (Marie-Anne). 2013. "Techno-discursivités natives sur Twitter. Une écologie du discours numérique ». Epistémè, 9, p. 139-176.

Poli (Marie-Sylvie) \& Ancel (Pascale). 2014. Exposer l'histoire contemporaine. Évaluation muséologique d'une exposition : "Spoliés ! L"aryanisation” économique en France (1940-1944). Paris : La Documentation française, coll. "Musées-Mondes".

Ricœur (Paul). 1983. Temps et récit. Paris : Seuil.

Riesman (David). 1964. La Foule solitaire. Paris : Arthaud.

Souchier (Emmanuel). 2012. " La "lettrure" à l'écran. Lire et écrire au regard des médias informatisés ». Communication \& Langages, 174, p. 85-108.

Spies (Virginie). «Twitter, Europe 1, Tous reporters ? ». Semioblog, 11 septembre 2011. Publication en ligne : http://semiologie-television.com/?p=3798". Consulté le 25.09.2014.

Stiegler (Bernard). 2013. "La société automatique », p. 84-98 in La Métamorphose numérique : Vers une société de la connaissance et de la coopération. Paris : Alternatives, coll. " Manifesté ».

Tobelem (Jean-Michel). 2010. Le Nouvel Âge des musées : Les institutions culturelles au défi de la gestion. Paris : Armand Colin, coll. « Sociétales ».

Wittgenstein (Ludwig). 1961. Tractatus logico-philosophicus, suivi de Investigations philosophiques. Paris : Gallimard, coll. « Tel », 109.

\section{Notes}

1 La Structure fédérative de recherche (SFR) Agorantic « Culture, Patrimoines, Sociétés numériques » de l'Université d'Avignon et des Pays de Vaucluse, Fédération de recherche (FR) CNRS 3621, est un exemple actif de ce type de projet. Publication en ligne : http://blogs.univavignon.fr/sfr-agorantic/. Consulté le 25.09.2014.

2 Ce thème, « Formes et modèles culturels », consacré à la genèse - sociale et technosémiotique - et à l'institutionnalisation des formes culturelles, est un des quatre domaines thématiques de l'UMR 8562 centre Norbert Elias. Publication en ligne : http://centre-norbert-elias.ehess.fr/ ! Consulté le 25.09.2014.

3 L'exposition Edward Hopper s'est tenue au Grand Palais à Paris du 10 octobre 2012 au 28 janvier 2013. Elle a été prolongée du 26 au 31 janvier 2013 ; durant cette prolongation l'exposition est restée ouverte de 9 heures à 23 heures ; puis jour et nuit du 1er au 3 février 2013, soit 62 heures d'ouverture sans interruption - gratuité étendue aux moins de 16 ans.

4 Le Festival de Cannes 2013 s'est tenu du 15 au 26 mai au palais des Festivals de Cannes. Le président du jury était cette année-là Steven Spielberg, et la maîtresse de cérémonie, Audrey Tautou. Pour la première fois, c'est sur Twitter que l'on a appris par Gilles Jacob, le président du conseil d'administration du Festival, que ce serait Uma Thurman qui remettrait la Palme d'or.

5 L'édifice abrite notamment la Nef, administrée par l'Établissement public du Grand Palais, et les Galeries nationales, gérées par la Réunion des musées nationaux. Publication en ligne : http://rmngp.fr/

6 Publication en ligne : http://www.grandpalais.fr/fr/evenement/edward-hopper. Consulté le 25.09.2014.

7 Ce travail de veille a été en partie réalisé avec les étudiants de première année du Master "Stratégies du développement culturel » de l'Université d'Avignon et des Pays de Vaucluse, spécialité « Médiation de la culture et des patrimoines » dans le cadre du séminaire " Métamorphose de l'écriture médiatique et médiation des savoirs ». 
8 Edward Hopper d'une fenêtre à l'autre, l'application iPad réalisée par la Réunion des musées nationaux (RMN) sous la direction de Didier Ottinger, commissaire de l'exposition, permet de visionner mais aussi d'écouter et de naviguer à l'intérieur de l'œuvre de l'artiste à partir de neuf fenêtres (neuf tableaux) qui traitent également de la vie de l'artiste, de ses aspirations, de ses partis pris esthétiques et picturaux. Pour chacune de ces « expositions-événements », le Grand Palais édite désormais une application qui n'est pas une visite virtuelle de l'exposition, mais plutôt un catalogue numérique réalisé sous la houlette du commissaire de l'exposition ; chaque application se caractérise par l'excellence de sa qualité scientifique et technologique, son niveau d'expertise en histoire de l'art.

9 Précisons que, " par réflexivité nous entendons plus spécifiquement ici la propriété de certaines situations de rendre disponible aux acteurs, en même temps qu'ils participent à des activités, une représentation évolutive de ce qu'ils sont en train de faire. Cet instrument des situations rend possible simultanément de faire et de se voir en train de faire » (Licoppe \& Dreatta, 2013 : 66).

10 Notons la facilité qu'offre Twitter grâce au hashtag : un mot précédé du signe " \# » comme " \#Cannes2013 » renvoie au même sujet auquel se réfèrent les twittos, qui échangent par conséquent à propos d'un sujet commun.

11 Gilles Jacob publie régulièrement sur Twitter son regard sur le Festival ainsi que des photographies, et le délégué artistique Thierry Frémaux utilise également le réseau social pour afficher son point de vue sur l'authenticité factuelle de ce qui se passe dans la manifestation qu'il anime.

12 Décrit ainsi par Thierry Frémaux sur la frontpage de présentation de son compte @THIERRYFREMAUX.

\section{Pour citer cet article}

Référence papier

Emmanuel Ethis et Marie-Sylvie Poli, « Hopper 2013/Cannes 2013 au prisme des écritures numériques », Culture \& Musées, 24 | 2014, 63-88.

Référence électronique

Emmanuel Ethis et Marie-Sylvie Poli, « Hopper 2013/Cannes 2013 au prisme des écritures numériques », Culture \& Musées [En ligne], 24 | 2014, mis en ligne le 19 juin 2018, consulté le 16 juin 2021. URL : http://journals.openedition.org/culturemusees/624 ; DOI :

https://doi.org/10.4000/culturemusees.624

\section{Cet article est cité par}

- Poli, Marie-Sylvie. (2018) Prescription culturelle. DOI: 10.4000/books.pressesenssib.9391

\section{Auteurs}

\section{Emmanuel Ethis}

Emmanuel Ethis est professeur des universités en Sciences de l'information et de la communication à l'université d'Avignon et des Pays de Vaucluse, dont il est également le président. Chercheur dans l'équipe Culture et Communication du centre Norbert Élias (UMR CNRS 8562), il préside le Haut Conseil de l'Éducation artistique et culturelle, l'Institut supérieur des techniques du spectacle et siège au conseil d'orientation scientifique du MUCEM et du musée du Quai Branly et est le représentant français de l'International Advisory Board of the Research Project Dynamics of World Cinema. Sociologue de la culture, Emmanuel Ethis consacre ses recherches à la sociologie du cinéma, à la réception des œuvres filmiques et à l'analyse des publics et des spectateurs de cinéma et des grands festivals (Cannes, Avignon...), ainsi qu'au parcours des comédiens saisis dans leur réalité anthropologique.

Ethis (Emmanuel). 2014. Sociologie du cinéma et de ses publics. Paris : Armand Colin [Troisième édition, revue et augmentée.]

Ethis (Emmanuel) \& Fabiani (Jean-Louis). 2014. « The Festival and the City: The Example of Avignon ». Eurozine.

Ethis (Emmanuel) \& Malinas (Damien). 2012. Les Films de campus, l'université au cinéma. Paris : Armand Colin.

Ethis (Emmanuel). 2012. La Petite Fabrique du spectateur. Être et devenir festivalier à Cannes et Avignon. Avignon : Les Éditions universitaires d'Avignon (EUA).

Ethis (Emmanuel). 2003. « Cannes hors projections » (direction du numéro) - Protée, revue de 
théories et pratiques sémiotiques, Université de Québec à Chicoutimi, volume 31, $\mathrm{n}^{\circ} 2$. emmanuel.ethis@univ-avignon.fr

Articles du même auteur

Avant-propos [Texte intégral]

Paru dans Culture \& Musées, Hors-série | 2013

Marie-Sylvie Poli

Marie-Sylvie Poli est professeure des universités en Sciences de l'information et de la communication à l'université d'Avignon et des Pays de vaucluse, chercheuse dans l'équipe

Culture et Communication du centre Norbert Élias (UMR CNRS 8562). Elle codirige la structure fédérative de recherche Agorantic « Culture, Patrimoines, Sociétés numériques » (FR CNRS 3621).

Elle s'intéresse surtout aux enjeux de la visite d'exposition chez les publics non experts. Ses recherches actuelles portent sur les nouvelles formes d'écriture de transmission des savoirs et leurs impacts sur la visite, depuis que le numérique et le participatif pénètrent tout le champ muséal.

Poli (Marie-Sylvie) \& Ancel (Pascale). 2014. Exposer l'histoire contemporaine. Évaluation muséologique d'une exposition : Spoliés ! L'« aryanisation » économique en France 1940-1944. Paris : La Documentation française, coll. « Musées-Mondes ».

Poli (Marie-Sylvie). 2013. «Éducation et musée » p. 165-189, in Culture \& Musées, 20 ans de muséologie, Arles : Éditions Actes Sud. Poli (Marie-Sylvie). 2013. « Les médiations du savoir au musée de la Cité nationale de l'histoire de l'immigration », p. 111-124 in Rouffineau (Gilles). (dir). Transmettre l'histoire : Contribution du design à la production des savoirs, Paris, B42Nalence, ESAD Grenoble-Valence.

marie-sylvie.poli@univ-avignon.fr

Articles du même auteur

La parade numérique du Musée du Désert [Texte intégral]

Paru dans Culture \& Musées, 37 | 2021

Hommage à Marie-Claire Habib [Texte intégral]

Paru dans Culture \& Musées, 36 | 2020

Visite du Musée Ghibli, à Tokyo [Texte intégral]

Paru dans Culture \& Musées, 36 | 2020

Artistes \& Robots [Texte intégral]

Grand Palais, Galeries nationales, Paris, 5 avril - 9 juillet 2018

Paru dans Culture \& Musées, 35 | 2020

Marie-Pierre Fourquet-Courbet \& Didier Courbet. Connectés et heureux ! Du stress digital au bien-être numérique [Texte intégral]

Paru dans Culture \& Musées, 35 | 2020

Cités millénaires. Voyage virtuel de Palmyre à Mossoul [Texte intégral] Institut du monde arabe (IMA), Paris, 10 octobre 2018 - 17 février 2019

Paru dans Culture \& Musées, 35 | 2020

Tous les textes...

\section{Droits d'auteur}

Culture \& Musées 